OPEN ACCESS

Edited by:

Xiaoya Chen,

Shanghai Institute of Plant Physiology

and Ecology, China

Reviewed by:

LiTian,

University of California, Davis, USA

Aixia Cheng,

Shandong University, China

${ }^{*}$ Correspondence:

Ying Wang

yingwang@wbgcas.cn

Specialty section:

This article was submitted to

Plant Metabolism

and Chemodiversity,

a section of the journal

Frontiers in Plant Science

Received: 13 May 2016

Accepted: 11 July 2016

Published: 21 July 2016

Citation:

Huang W, Khaldun ABM, Chen J, Zhang $C, L V H$, Yuan $L$ and Wang $Y$

(2016) A R2R3-MYB Transcription

Factor Regulates the Flavonol Biosynthetic Pathway in a Traditional Chinese Medicinal Plant, Epimedium sagittatum. Front. Plant Sci. 7:1089.

doi: 10.3389/fpls.2016.01089

\section{A R2R3-MYB Transcription Factor Regulates the Flavonol Biosynthetic Pathway in a Traditional Chinese Medicinal Plant, Epimedium sagittatum}

\author{
Wenjun Huang', A. B. M. Khaldun', Jianjun Chen', Chanjuan Zhang', Haiyan Lv', \\ Ling Yuan ${ }^{3}$ and Ying Wang ${ }^{1 *}$
}

'Key Laboratory of Plant Germplasm Enhancement and Specialty Agriculture, Wuhan Botanical Garden - Chinese Academy of Sciences, Wuhan, China, ${ }^{2}$ Key Laboratory of Oil Crop Biology of the Ministry of Agriculture, Oil Crops Research Institute of the Chinese Academy of Agriculture Sciences, Wuhan, China, ${ }^{3}$ Department of Plant and Soil Sciences, University of Kentucky, Lexington, KY, USA

Flavonols as plant secondary metabolites with vital roles in plant development and defense against UV light, have been demonstrated to be the main bioactive components (BCs) in the genus Epimedium plants, several species of which are used as materials for Herba Epimedii, an important traditional Chinese medicine. The flavonol biosynthetic pathway genes had been already isolated from Epimedium sagittatum, but a R2R3MYB transcription factor regulating the flavonol synthesis has not been functionally characterized so far in Epimedium plants. In this study, we isolated and characterized the R2R3-MYB transcription factor ESMYBF1 involved in regulation of the flavonol biosynthetic pathway from $E$. sagittatum. Sequence analysis indicated that EsMYBF1 belongs to the subgroup 7 of R2R3-MYB family which contains the flavonol-specific MYB regulators identified to date. Transient reporter assay showed that EsMYBF1 strongly activated the promoters of ESF3H (flavanone 3-hydroxylase) and EsFLS (flavonol synthase), but not the promoters of EsDFRs (dihydroflavonol 4-reductase) and ESANS (anthocyanidin synthase) in transiently transformed Nicotiana benthamiana leaves. Both yeast two-hybrid assay and transient reporter assay validated ESMYBF1 to be independent of EsTT8, or AtTT8 bHLH regulators of the flavonoid pathway as cofactors. Ectopic expression of ESMYBF1 in transgenic tobacco resulted in the increased flavonol content and the decreased anthocyanin content in flowers. Correspondingly, the structural genes involved in flavonol synthesis were upregulated in the EsMYBF1 overexpression lines, including $\mathrm{NtCHS}$ (chalcone synthase), $\mathrm{NtCHI}$ (chalcone isomerase), NtF3H and NtFLS, whereas the late biosynthetic genes of the anthocyanin pathway (NtDFR and NtANS) were remarkably downregulated, compared to the controls. These results suggest that ESMYBF1 is a flavonol-specific R2R3-MYB regulator, and involved in regulation of the biosynthesis of the flavonol-derived BCs in E. sagittatum. Thus, identification and functional characterization of ESMYBF1 provide insight into understanding the biosynthesis and regulation of the flavonol-derived BCs in Epimedium plants, and also provide an effective tool gene for genetic manipulation to improve the flavonol synthesis.

Keywords: Epimedium, flavonoid, flavonol, MYB, transcription factor 


\section{INTRODUCTION}

Flavonoids are a large class of plant secondary metabolites, and play diverse roles in plant development and defense. They are derived from phenylalanine and divided into three major classes: anthocyanins, proanthocyanidins (PAs), and flavonols. For plant itself, the most prominent function of flavonols is to protect plant against UV light damage. While for human health, flavonols are shown to possess antiinflammatory, antioxidant, and antiproliferative capacities (Havsteen, 2002; Yao et al., 2004). Flavonoids, including flavonols attract a growing number of interests from public due to their significant benefits on human health.

The medicinal plants are generally known for the abundance of certain bioactive compounds, such as flavonoids, alkaloids, and terpenoids. For an example, Herba Epimedii, an important traditional Chinese medicine, is featured by icariin, a C8prenylated flavonol glycoside (Ma et al., 2011). Herba Epimedii is prepared from the dried aerial parts of Epimedium species in the family Berberidaceae (Li et al., 2005). Herba Epimedii, traditionally used as a kidney tonic and antirheumatic medicinal herb for more than 2000 years, is also currently used for treating many other disease, including sexual dysfunction, osteoporosis, diabetics, cardiovascular disease, tumors, and immunoregulation (Ma et al., 2011; Li et al., 2015a; Jiang et al., 2016). Flavonoids, especially C8-prenylated flavonol glycosides have been demonstrated to be the main bioactive components (BCs) in Epimedium plants, such as icariin which is one of the most studied BCs with extensive therapeutic capacities including osteoprotective effect, cardiovascular protective effect, anti-cancer effect, and anti-inflammation effect (Ma et al., 2011; Jiang et al., 2015; Li et al., 2015a). Although the main flavonolderived BCs have been phytochemically and pharmacologically well-characterized, and their molecular biosynthetic pathways have been clarified recently (Zeng et al., 2013; Huang et al., 2015), but the studies of regulation of the synthesis of these flavonols in Epimedium plants are scarcely reported.

As we know, flavonol biosynthesis is one of the branches of the flavonoid biosynthetic pathway. The flavonoid biosynthetic pathway has been extensively studied in several model plant species, including Arabidopsis, petunia, maize, and grape (Winkel-Shirley, 2001; Koes et al., 2005). Recently, in Epimedium plants, the flavonoid biosynthetic pathway has also been elucidated, and most of structural genes of this pathway has been isolated (Zeng et al., 2013; Huang et al., 2015). Moreover, it is well-established that the flavonoid biosynthetic pathway is predominantly regulated by $M Y B, b H L H$, and $W D 40$ regulatory genes at the transcriptional level (Ramsay and Glover, 2005; Hichri et al., 2011). An increasing number of MYB TFs regulating the flavonoid biosynthetic pathway have been identified from many plant species, especially Arabidopsis, maize, petunia, snapdragon, and grape with abundant functionally characterized $M Y B$ regulators (Liu et al., 2015). Each specific branch of the flavonoid pathway in both Arabidopsis and grape is generally regulated by a different $M Y B$ regulator. There are AtPAP1 and AtPAP2 from Arabidopsis (Borevitz et al., 2000), VvMYBA1 and VvMYBA2 (Kobayashi et al., 2004; Walker et al., 2007) from grape identified to regulate anthocyanin biosynthetic branch, Arabidopsis AtTT2 (Nesi et al., 2001) and grape VvMYBPA1, VvMYBPA2 (Bogs et al., 2007; Terrier et al., 2009) identified to regulate proanthocyanidin (PA) biosynthetic branch, while AtMYB12/AtMYB11/AtMYB111 (Mehrtens et al., 2005; Stracke et al., 2007) and VvMYBF1 (Czemmel et al., 2009) identified to regulate flavonol biosynthetic branch. Corresponding, in Epimedium plants, two R2R3-MYB regulators, EsMYBA1 and EsAN2 were recently characterized to regulate anthocyanin biosynthetic pathway in a different tissue-specific manner (Huang et al., 2013, 2016). The correlation of gene-to-metabolite during leaf developmental stages of Epimedium sagittatum has been systematically analyzed in the survey (Huang et al., 2015). A R2R3-MYB TF out of a dozen of MYB TFs studied was found to basically correlate with the accumulation patterns of the main four BCs (epimedin A, B, C and icariin) and coexpress with several the flavonol biosynthetic pathway genes. The primary results suggest that this $M Y B$ gene is probably involved in regulating the flavonol pathway (Huang et al., 2015). Due to the high level of sequence similarity with grape $V v M Y B F 1$, this $M Y B$ gene from E. sagittatum was designated as EsMYBF1 gene. However, the functional characterization of EsMYBF1 gene is still far from being clarified.

In regulation of the anthocyanin and PA biosynthetic pathways, MYB TFs generally interact with bHLH TFs and WD40 proteins to form a MYB-bHLH-WD40 (MBW) complex (Xu et al., 2015). For example in Arabidopsis, AtPAP1/AtPAP2AtGL3/AtEGL3-AtTTG1 complex regulates anthocyanin synthesis (Gonzalez et al., 2008). However, in regulation of the flavonol biosynthetic pathway, R2R3-MYB regulators identified to date are found to be independent of $b H L H$ cofactor. Three R2R3-MYB members (AtMYB12/11/111) of Arabidopsis and $V v M Y B F 1$ of grape function as a activator of flavonol synthesis without binding a $b H L H$ partner (Mehrtens et al., 2005; Stracke et al., 2007; Czemmel et al., 2009), probably because they don't have the conserved $[\mathrm{DE}] \mathrm{Lx}_{2}[\mathrm{RK}] \mathrm{x}_{3} \mathrm{Lx}_{6} \mathrm{Lx}_{3} \mathrm{R}$ region described previously by Zimmermann et al. (2004) for interacting with bHLH regulators, which is generally required for the $M Y B$ regulators of both anthocyanin and PA biosynthesis. Moreover, these flavonol-specific MYB regulators can activate the promoters of target genes involved in flavonol biosynthesis, including $\mathrm{CHS}$, CHI, F3H, and FLS (Mehrtens et al., 2005; Czemmel et al., 2009). Therefore, it is worthy to investigate whether or not the $E s M Y B F 1$ gene is independent of $b H L H$ cofactor and specifically regulates the flavonol biosynthetic pathway genes.

Majority of the flavonoid biosynthetic pathway genes in Epimedium have been isolated, but two $M Y B$ regulators of the flavonoid pathway reported to date are just confined to the anthocyanin synthesis, including EsMYBA1 and EsAN2 regulators (Huang et al., 2013, 2016). Material supply of Herba Epimedii mostly depends on wild resource of Epimedium plants, and certain species have been threatened with risk of extinction due to over-harvesting (Li et al., 2005). The flavonol-derived BCs accumulate lowly in Epimedium plants, and the overexpression of a flavonol-specific $M Y B$ regulator in transgenic plants is a powerful alternative way to improve the biosynthesis and accumulation of the desired flavonols. However, the putative 
EsMYBF1 TF regulating flavonol biosynthesis has not been functionally characterized so far in Epimedium or any other medicinal plants until now. Herein, the putative flavonol-specific ESMYBF1 regulator would be functionally characterized indepthly. The results indicate that EsMYBF1 is mainly expressed in leaves, and correlates with the accumulation of the main BCs during leaf development. EsMYBF1 strongly activates the promoters of EsF3H and EsFLS genes involved in the flavonol biosynthesis rather than the promoters of EsDFRs and EsANS leading to the anthocyanin synthesis. Overexpression of EsMYBF1 in tobacco results in the enhanced flavonol content and the reduced anthocyanin content, through upregulating the flavonol pathway genes and downregulating the late anthocyanin biosynthetic genes. The functional characterization of EsMYBF1 regulator of flavonol synthesis makes a further step forward in understanding the regulatory mechanism of the flavonolderived BCs biosynthesis in Epimedium plants, and also provides an effective tool gene for genetic manipulation to create new cultivars.

\section{RESULTS}

\section{Sequence Analysis and Expression Pattern of EsMYBF1 Gene}

The full-length cDNA clone of EsMYBF1 was successfully obtained from E. sagittatum leaves using RACE (Rapid Amplification of cDNA Ends) method. EsMYBF1 was predicted to have an entire ORF of 1146 bp length, encoding a R2R3-MYB protein of 381 amino acid residues in length. Sequence analysis revealed that EsMYBF1 contains the R2 and R3 MYB DNAbinding domains in its $\mathrm{N}$-terminus (Figure 1A). The redefined SG7 motif $([\mathrm{K} / \mathrm{R}][\mathrm{R} / \mathrm{x}][\mathrm{R} / \mathrm{K}] \mathrm{xGRT}[\mathrm{S} / \mathrm{x}][\mathrm{R} / \mathrm{G}] \mathrm{xx}[\mathrm{M} / \mathrm{x}] \mathrm{K})$ characteristic of flavonol regulators of Arabidopsis and Vitis vinifera (Czemmel et al., 2009) was also found to be present in the C-terminus of VvMYBF1 (Figure 1A). Besides, EsMYBF1 also contains a similar counterpart (WLEE) of the SG7-2 motif $([\mathrm{W} / \mathrm{x}][\mathrm{L} / \mathrm{x}] \mathrm{LS})$ identified previously (Czemmel et al., 2009) in the C-terminus. Additionally, it is worthy to notice that the $\mathrm{R} 2 \mathrm{R} 3$ repeat region of EsMYBF1 does not contain the motif $[\mathrm{D} / \mathrm{E}] \mathrm{Lx}_{2}[\mathrm{R} / \mathrm{K}] \mathrm{x}_{3} \mathrm{Lx}_{6} \mathrm{Lx}_{3} \mathrm{R}$ for interaction with $\mathrm{bHLH}$ partners (Zimmermann et al., 2004). Sequence similarity between MYB proteins is generally confined to the R2 and R3 repeats. EsMYBF1 showed high identity with other MYB regulators within the R2 and R3 MYB domains, such as $87 \%$ identity with apple MdMYB22 and 82\% identity with Arabidopsis AtMYB12. However, within the overall protein sequence, EsMYBF1 showed only 44\% identity to grape VvMYBF1, and $40 \%$ identity to Arabidopsis AtMYB111. Phylogenetic analysis indicated that EsMYBF1 was clustered with other flavonol MYB regulators together into the flavonol clade, and was closely related to Arabidopsis AtMYB111 regulator of flavonol synthesis (Figure 1B).

The genomic sequence of EsMYBF1 was also isolated from genomic DNA of E. sagittatum. Alignment between the genomic and CDNA sequences showed that EsMYBF1 consisted of three exons and two introns. These two introns were located into the
R2 and R3 MYB domain, respectively (Figure 2). Moreover, the intron insertion places are highly conserved (data not shown). Additionally, to analyze the expression pattern of EsMYBF1 in various tissues of Epimedium, qPCR assay was carried out. The results showed that EsMYBF1 is mainly expressed in leaves, and very lowly expressed in other tissues (Figure 3).

\section{EsMYBF1 Is Independent of EsTT8 and AtTT8 bHLH Cofactors}

It is previously reported that the flavonol-specific $M Y B$ regulators are independent of $b H L H$ cofactors. In order to validate whether or not EsMYBF1 also has this characterization, $\mathrm{Y} 2 \mathrm{H}$ assay of EsMYBF1 with AtTT8 or EsTT8 bHLH regulators was performed. The results showed that a total of eight combinations was able to grow on the double SD medium, but only two combinations, including the positive control (pGBKT7-53+ pGADT7-T) and the EsMYBF1-BD + pGADT7 constructs, grew on the quadruple SD medium (Figure 4). These results suggest that EsMYBF1 has a strong autoactivation activity, and does not interact with AtTT8 or EsTT8 bHLH regulators. This conclusion was further supported by the formation of blue colonies in the corresponding yeast cells through $\beta$-galactosidase assay (Figure 4). Finally, EsMYBF1 is demonstrated to be independent of $b H L H$ cofactor.

\section{EsMYBF1 Activates Promoters of the Flavonol Pathway Genes}

To identify the target genes of the flavonoid pathway by EsMYBF1 TF, the dual luciferase assay system in transiently transformed Nicotiana benthamiana leaves was carried out. EsCHS and EsF3H as general flavonoid pathway genes are involved in the synthesis of flavonols, anthocyanins and PAs. Meanwhile, EsDFRs and EsANS are required for anthocyanin synthesis, whereas EsFLS as the flavonol specific branch point gene is specifically required for flavonol synthesis. Therefore, the promoters of these structural genes were chosen as potential targets of ESMYBF1 transcription activation. The results showed that EsMYBF1 strongly activated the promoters of both $E s F 3 H$ and EsFLS, and slightly activated the promoter of EsCHS closely twofold, compared to the corresponding controls which only consisted of promoters without $M Y B$ TFs. Similarly, AtMYB12 regulator of flavonol pathway as a positive control, also strongly activated the promoters of EsF3H and EsFLS, and slightly activated the promoter of EsCHS approximately twofold (Figure 5). However, the addition of EsTT8 or AtTT8, a bHLH factor generally required as a cofactor for $M Y B$ transcription activator of flavonoid pathway, decreased the activation potential of $E s M Y B F 1$ on the $E s F 3 H$ promoter, or approximately equalized the activation of ESMYBF1 on the promoters of both EsFLS and $E s C H S$, compared with EsMYBF1 alone (Figure 5). In addition, the relative values of transcription activation of EsMYBF1 alone or combined with EsTT8 or AtTT8 bHLH regulators on the promoters of EsDFR1, EsDFR2 and EsANS were approximately equal to that of the empty controls (promoter without MYB TF), and all these relative ratios were very close to zero (Figure 5), suggesting that EsMYBF1 cannot activate the promoters of 
A

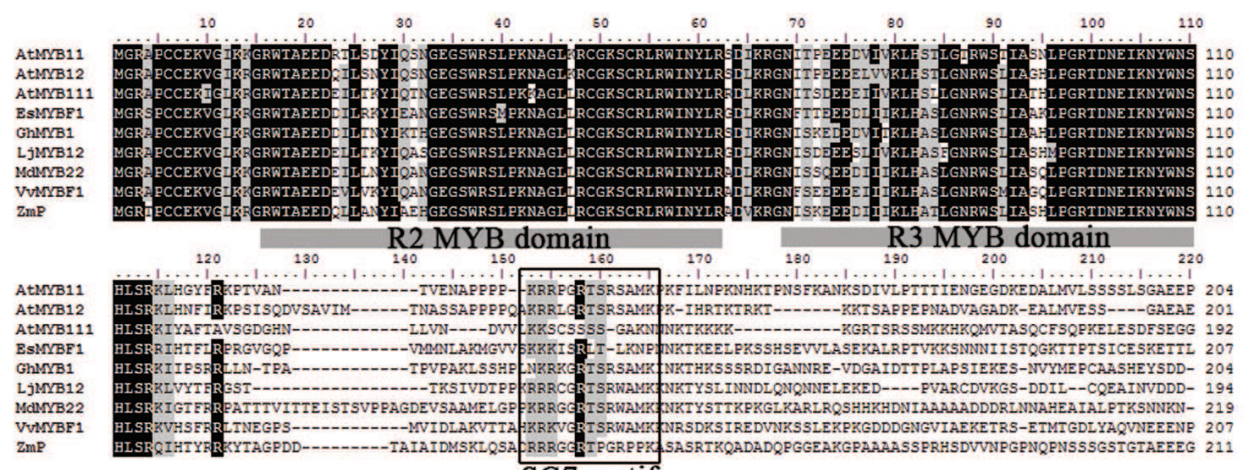

$\mathrm{ZmP}$

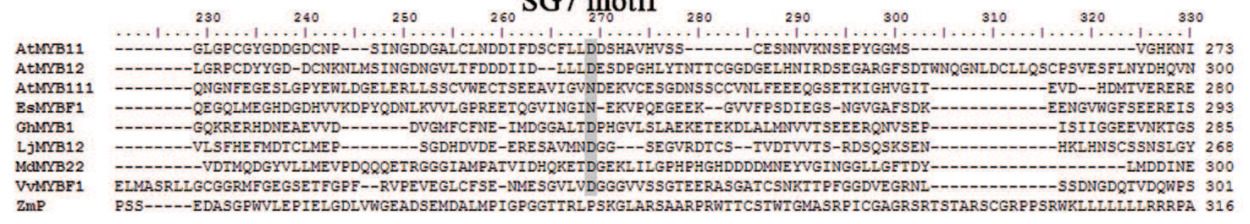

$\mathrm{ZmP}$

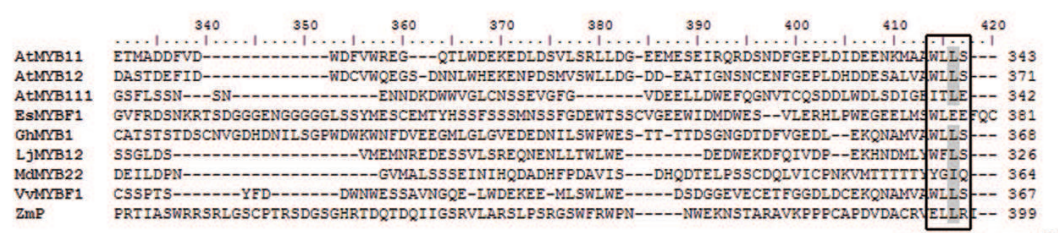

SG7-2 motif

B

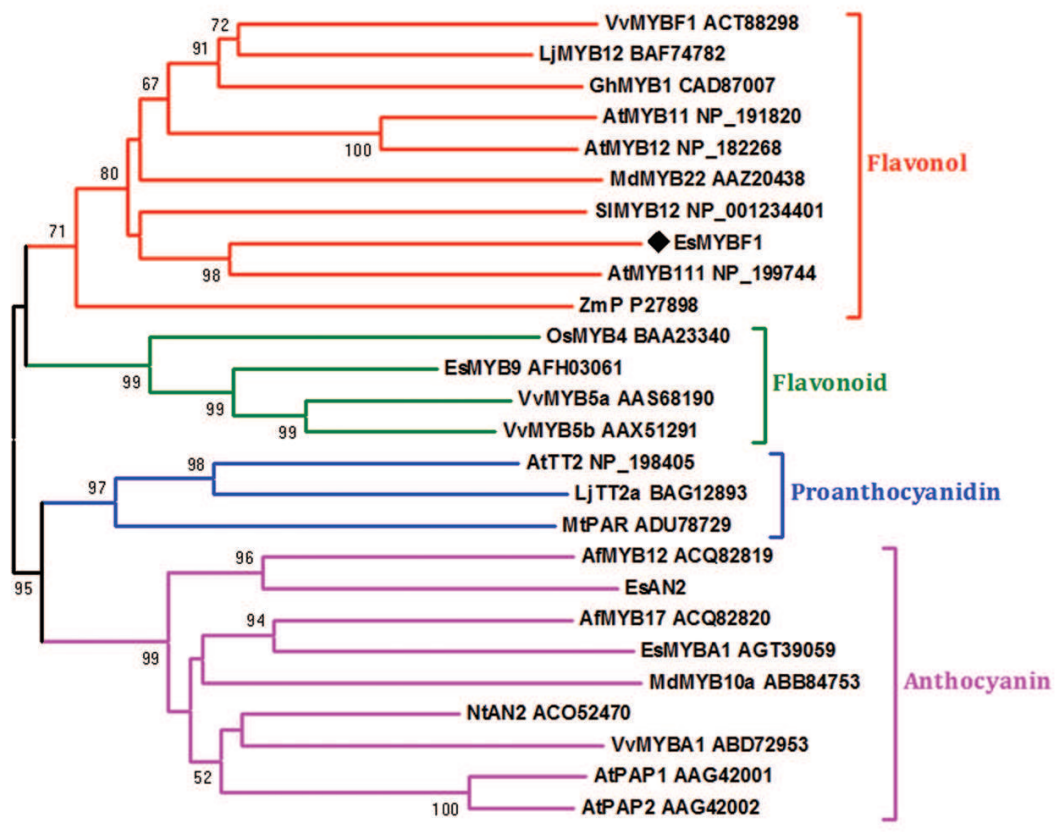

0.1

FIGURE 1 | Multiple alignment and phylogenetic analysis of EsMYBF1 and other plant R2R3-MYB proteins known to regulate the flavonoid biosynthetic pathway. (A) Alignment of deduced amino acid sequences of EsMYBF1 and selected R2R3-MYB regulators of flavonol biosynthesis from other plant species. Identical amino acid residues are shaded in black, and similar in gray. The R2 and R3 MYB domains shown refer to two repeats of the MYB DNA-binding domain of MYB proteins. Two conserved motifs, SG7 and SG7-2 motifs are boxed. (B) Phylogenetic analysis of EsMYBF1 and selected R2R3-MYB proteins from other plant species. The phylogenetic tree is constructed using the neighbor-joining method by MEGA 5 software. The scale bar represents 0.1 substitution per site, and the numbers next to nodes are bootstrap values from 1,000 replicates. Bootstrap values less than 50 are not shown. EsMYBF1 is indicated as a diamond. Functions of most of the R2R3-MYB proteins are indicated. All R2R3-MYB protein sequences are retrieved from GenBank database, and their accession number are embedded in the diagram. Plant species are as follows: Aquilegia formosa, Arabidopsis thaliana, Epimedium sagittatum, Gerbera hybrid, Lotus japonicus, Malus $x$ domestica, Medicago truncatula, Nicotiana tabacum, Oryza sativa, Solanum lycopersicum, Vitis vinifera, Zea mays. 


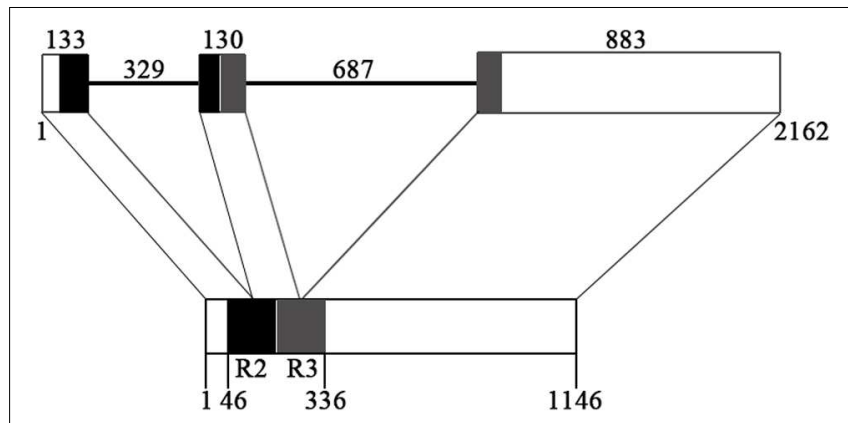

FIGURE 2 | Genomic structure of EsMYBF1 gene. The R2 and R3 MYB DNA-binding domains are shown as black and gray blocks, respectively. The exons are shown as blocks and the introns as lines. The diagram top represents the genomic sequence, and the numbers located above show the length of exons and introns. The bottom of diagram means the cDNA sequence, and the numbers located below show the distance from the start codon of ESMYBF1 gene.

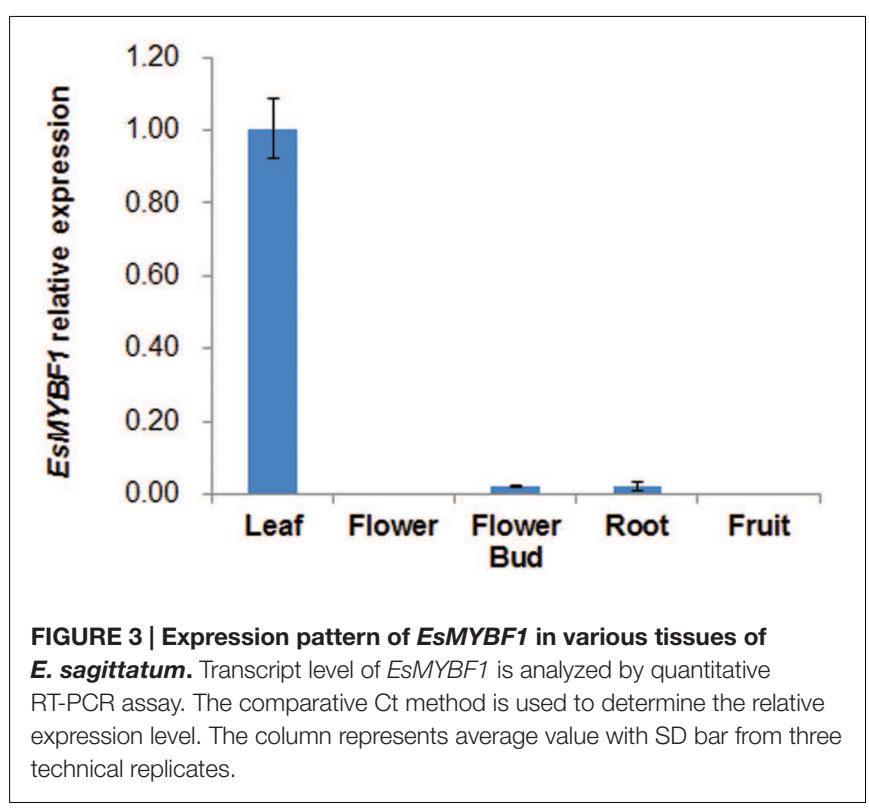

EsDFRs and EsANS. In summary, these results suggest that $E s M Y B F 1$ is a specific regulator of flavonol synthesis, potentially regulating the EsF3H and EsFLS genes, and is independent of EsTT8 or AtTT8 bHLH cofactors.

\section{Overexpression of EsMYBF1 in Tobacco Affects the Accumulation of Flavonoids}

To characterize the function of EsMYBF1, overexpression of $E s M Y B F 1$ in tobacco was carried out. The results showed that flower color of transgenic tobacco carrying EsMYBF1 turned from rosy red to light pink (Figure 6A). Among the three overexpression lines (F33, F38, and F40), flower color change of F40 lines was most strong. Corresponding to the phenotypic changes, flavonoid contents, including total anthocyanins and flavonols in transgenic tobacco flowers were measured. The results indicated that total anthocyanin content was significantly decreased in all three overexpression lines, compared to the controls (Figure 6B). However, flavonol content (kaempferol and quercetin) was significantly increased by twofold in all three overexpression lines, compared to the controls (Figure 6C). These results suggest ectopic expression of EsMYBF1 in tobacco directed the metabolic flux from anthocyanin pathway to flavonol pathway.

\section{Overexpression of EsMYBF1 in Tobacco Affects the Expression Levels of Flavonoid Pathway Genes}

The effect of EsMYBF1 overexpression on the expression levels of flavonoid pathway genes in tobacco flowers was analyzed by qPCR assay. The presence of EsMYBF1 in transgenic tobacco was firstly confirmed by RT-PCR assay (data not shown). The qPCR results showed that five structural genes, including $\mathrm{NtCHS}$, $\mathrm{NtCHI}, \mathrm{NtF} 3 \mathrm{H}, \mathrm{NtF3^{ \prime } H}$ and $\mathrm{NtFLS}$, were remarkably upregulated in two overexpression lines (F33 and F40), but NtDFR and NtANS was remarkably downregulated in two overexpression lines (F33 and F40), especially in F40 line, compared to the controls (Figure 7). Moreover, $\mathrm{NtF3H}$ was most highly increased, followed by NtCHI and NtFLS, particularly in F33 and F40 lines. It is notable that in $\mathrm{F} 38$ lines the change extent of gene expression was obviously lower than that in other two overexpression lines (Figure 7).

\section{DISCUSSION}

The functional similarity of R2R3-MYB TFs can be predicted by their structural similarity. The isolation of Arabidopsis AtMYB12 was just derived from sequence similarity analysis with maize $\mathrm{P}$ regulator (Mehrtens et al., 2005), and VvMYBF1 isolation was from AtMYB12 homolog (Czemmel et al., 2009). In this study, the putative ESMYBF1 regulator of flavonol pathway was also derived from these two AtMYB12 and $V v M Y B F 1$ homologs. EsMYBF1 shares the highly conserved R2 and R3 MYB domains in the $\mathrm{N}$-terminus, and the redefined SG7 and SG7-2 motifs (Czemmel et al., 2009) in the diverse C-terminus with other flavonolspecific MYB regulators (Figure 1A). Additionally, the R2 and R3 domains of EsMYBF1 does not contain the conserved bHLH interaction motif identified previously (Zimmermann et al., 2004), suggesting that EsMYBF1 is bHLH cofactor independent. This cofactor independency is generally restricted to the MYB factors of the flavonol clade (Figure 1B). In consideration of the high structural similarity of EsMYBF1 and members of the MYB subgroup 7, EsMYBF1 is predicted to be a putative flavonol regulator of MYB subgroup 7.

As we know, the expression of the $M Y B$ regulator of flavonol pathway correlates well with the flavonol synthesis and accumulation, such as Arabidopsis AtMYB12 and grapevine VvMYBF1 (Mehrtens et al., 2005; Czemmel et al., 2009). It was previously reported that the expression pattern of EsMYBF1 basically correlates with the accumulation pattern of the four flavonol-derived BCs during leaf development of Epimedium (Huang et al., 2015). Moreover, our results indicated that EsMYBF1 is most abundantly expressed in leaves (Figure 3), 

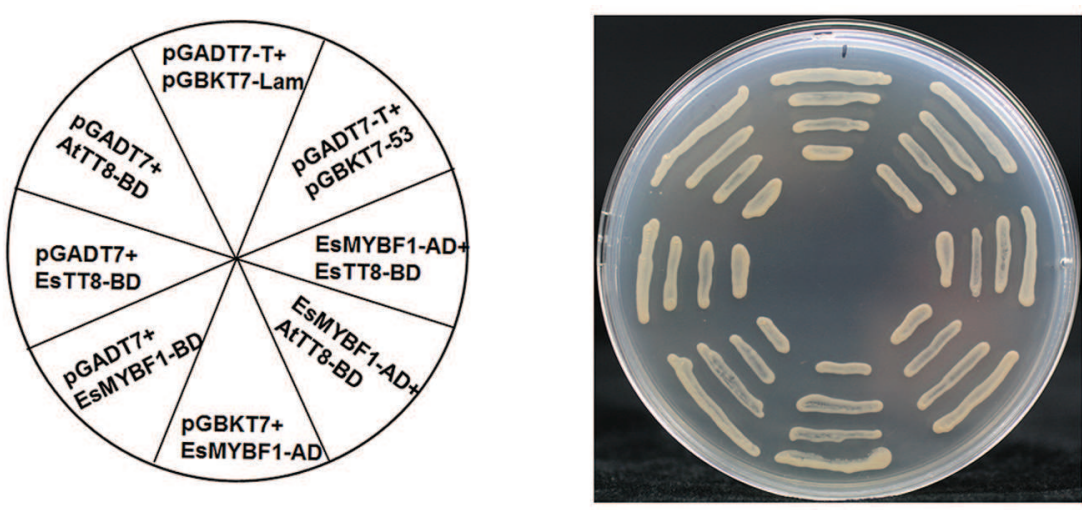

$\mathrm{SD} /$-Leu/-Trp

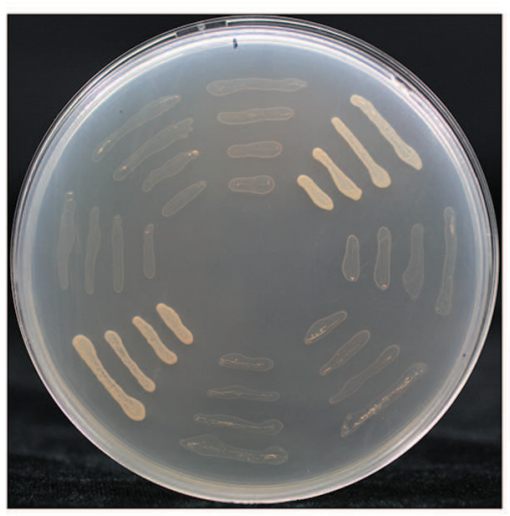

SD/-Leu/-Trp/-Ade/-His

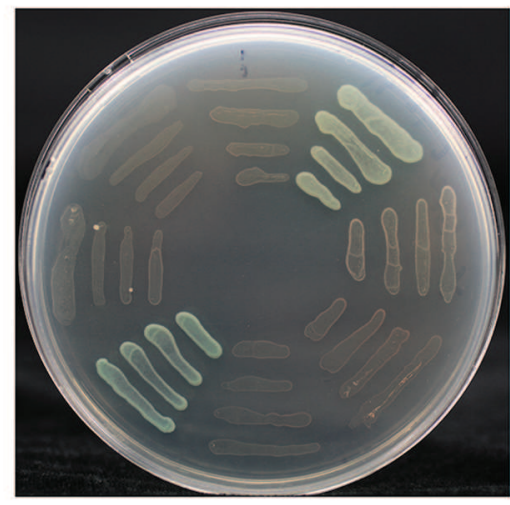

$\beta$-Galactosidase assay

FIGURE 4 | Yeast two-hybrid assay of EsMYBF1 with two bHLH regulators of flavonoid pathway. Two bHLH regulators involved in regulation of flavonoid biosynthetic pathway, EsTT8 from E. sagittatum and AtTT8 from A. thaliana are used for yeast two-hybrid assay. Transformed yeast cells containing pGBKT7-53+pGADT7-T, pGBKT7-Lam+pGADT7-T constructs are used as a positive and a negative control, respectively.

where the main flavonol-derived BCs accumulate largely. These results suggest that $E S M Y B F 1$ is involved in the developmental regulation of the flavonol-derived BCs in Epimedium leaves.

Both AtMYB12 and VvMYBF1 were previously reported to activate the flavonol pathway genes without the need of a bHLH cofactor (Mehrtens et al., 2005; Czemmel et al., 2009), probably because they do not contain the bHLH interaction motif in the R2R3 MYB domain. Similarly, the absence of the bHLH interaction motif in EsMYBF1 protein also contributed to that EsMYBF1 is independent of bHLH cofactors. It was demonstrated firstly that EsMYBF1 does not interact with the two bHLH regulators of flavonoid pathway in yeast two-hybrid assay, including EsTT8 from E. sagittatum and AtTT8 from Arabidopsis (Figure 4). Sequentially, the inclusion of EsTT8 or AtTT8 bHLH regulator was not required for transcription activation by EsMYBF1 in the transient reporter assay, and the presence of the $b H L H$ actually slightly diminished activation (Figure 5). Although the promoter of EsCHS was slightly activated by EsMYBF1, the promoters of EsF3H and EsFLS was strongly activated by EsMYBF1 (Figure 5), suggesting that $E s M Y B F 1$ is able to control the entire pathway leading to flavonol synthesis. Similar results were previously reported for the control of flavonol synthesis by AtMYB12/11/111 of Arabidopsis and VvMYBF1 of grapevine (Stracke et al., 2007; Czemmel et al., 2009). In addition, the CHS enzyme is encoded by a small of gene family, and three copies of CHS in E. sagittatum were found (Zeng et al., 2013). Therefore, the EsCHS member chose for the transient reporter assay may be not the real target of EsMYBF1 TF, because AtMYB12 regulator as a positive control still does not strongly induce the promoter of this EsCHS (Figure 5). It is noticeable that EsDFRs and EsANS genes encode enzymes responsible for anthocyanin and PAs synthesis, but their promoters were not activated by EsMYBF1 in the transient reporter assay (Figure 5). These results further confirmed that $E s M Y B F 1$ is a flavonol-specific MYB regulator, just like AtMYB12 and $V v M Y B F 1$ regulators. In summary, EsMYBF1 specifically activates the flavonol pathway genes without the need of a $b H L H$ cofactor.

The function of $M Y B$ regulating the flavonol synthesis is generally highly conserved among the different plant species. Similar results were obtained for the flavonol-specific $M Y B$ regulators when overexpressed in a heterologous system, such 


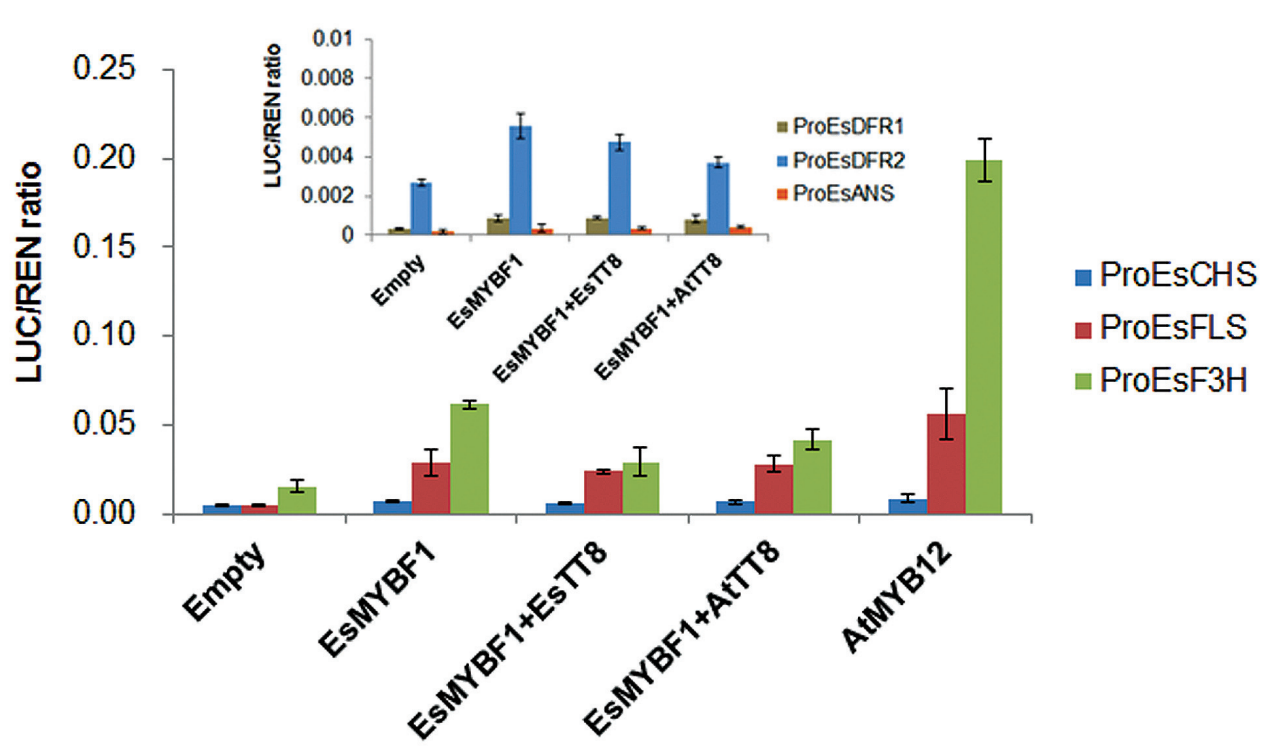

FIGURE 5 | Transcription activity assay of ESMYBF1 against promoters of flavonoid-related genes with and without bHLH regulators in transiently transfected Nicotiana benthamiana leaves. Six promoters of flavonoid biosynthetic genes from E. sagittatum are used for dual luciferase assay, including ProEsCHS, ProEsF3H, ProEsFLS, ProEsDFR1, ProEsDFR2, and ProEsANS. Two bHLH transcription factors involved in regulation of the flavonoid biosynthetic pathway, ESTT8 from E. sagittatum and AtTT8 from A. thaliana are also used as cofactors. Transformed leaves carrying only the promoter-LUC reporter construct without the transcription factors-containing effectors are used as controls. The columns represent average values with SD bar from four biological replicates for each treatment.

as AtMYB12 overexpression in tobacco (Misra et al., 2010) or in tomato (Luo et al., 2008), and AtMYB11 overexpression in tobacco and tomato (Li et al., 2015b). Ectopic expressions of these $M Y B$ regulators in transgenic tobacco or tomato can modulate the phenylpropanoid pathway genes and then the flavonoid accumulation, in particular upregulate the flavonol pathway genes and enhance the flavonol accumulation. Corresponding, overexpression of EsMYBF1 in tobacco resulted in the elevated expression of the flavonol pathway genes and the enhanced accumulation of flavonols (Figures 6 and 7). Global gene expression analysis for AtMYB12 overexpression in tobacco or in tomato indicated that AtMYB12 can modulate numbers of molecular processes, including aromatic amino acid biosynthesis, phytohormone signaling, stress responses and phenylpropanoid biosynthesis, in addition to the flavonol biosynthetic pathway (Luo et al., 2008; Misra et al., 2010; Pandey et al., 2015a). A previous study showed that the pathway genes leading to flavonol synthesis are upregulated in transgenic tobacco by ectopic expression of AtMYB12, including PAL (phenylalanine ammonia lyase), $\mathrm{C} 4 \mathrm{H}$ (cinnamate 4-hydroxylase), $\mathrm{CHS}, \mathrm{CHI}$, $F 3 H$, and FLS genes (Misra et al., 2010). The ectopic expression of EsMYBF1 in tobacco also produced a similar results (Figure 7). Moreover, three genes $\mathrm{NtF} 3 \mathrm{H}, \mathrm{NtCHI}$ and $\mathrm{NtFLS}$ involved in flavonol synthesis were more considerably upregulated than other upregulated genes of flavonoid pathway (Figure 7). Similarly, tomato $C H S, F L S$, and $F 3 H$ genes were most strongly induced by AtMYB11 or AtMYB12 overexpression in transgenic tomato (Li et al., 2015b; Pandey et al., 2015a). These target genes for EsMYBF1 TF regulation are indispensable for the biosynthesis of flavonols. In addition, due to the direction of the metabolic flux from anthocyanin to flavonol pathway, the accumulation of anthocyanin in transgenic tobacco flowers was remarkably decreased (Figure 6B). Similar results were also reported for AtMYB12 overexpression in tobacco and tomato (Luo et al., 2008; Pandey et al., 2015a), and AtMYB11 overexpression in tobacco (Pandey et al., 2015b). This reduction of anthocyanin accumulation can be explained by the significant downregulation of NtDFR and NtANS genes which are required for anthocyanin synthesis. Finally, these results proved that EsMYBF1 is a functional regulator of flavonol synthesis.

\section{CONCLUSION}

A R2R3-MYB TF, EsMYBF1 was isolated from E. sagittatum and functionally characterized. The present work demonstrates that $E s M Y B F 1$ is a flavonol-specific $M Y B$ regulator which can control the flavonol pathway genes. Preferential expression in leaves of ESMYBF1 and its correlation with the accumulation patterns of the main BCs during leaf development suggest that $E S M Y B F 1$ is involved in the developmental regulation of the biosynthesis of the flavonol-derived BCs in Epimedium leaves. Additionally, EsMYBF1 functions as a regulator of flavonol synthesis without the $b H L H$ cofactor. The functional characterization of EsMYBF1 not only provides insight into understanding the regulation of the biosynthesis of the flavonol-derived BCs in Epimedium plants, and also provides a potential effective TF for genetic manipulation to improve the accumulation of flavonols in the transgenic plants. 


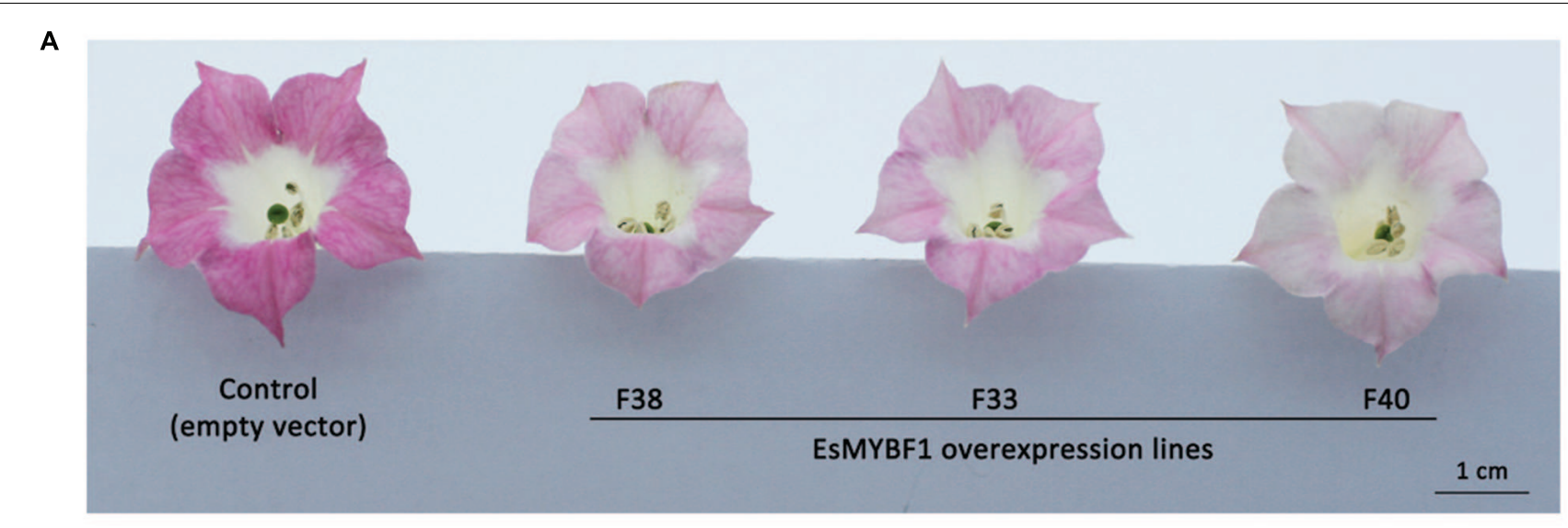

B

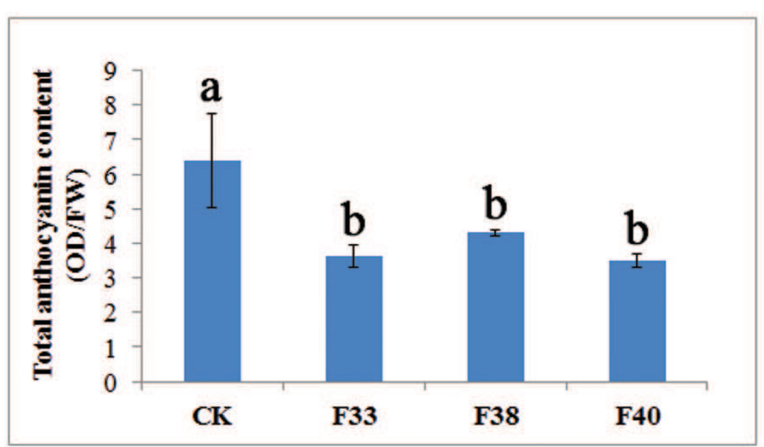

C

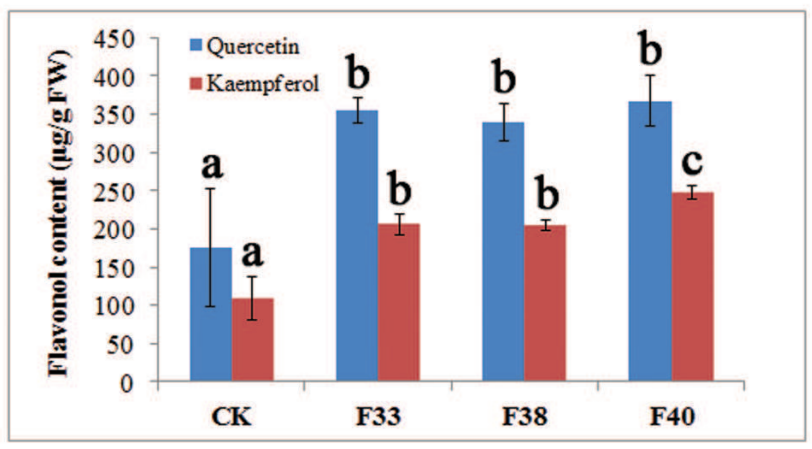

FIGURE 6 | Phenotype observation and flavonoid content measurement in transgenic tobacco overexpressing EsMYBF1 gene. Three independent transgenic tobacco lines carrying ESMYBF1 (F33, F38, F40) and the transgenic tobacco carrying the empty vector as control plants are used. Phenotypic changes observation (A), and total anthocyanin content measurement (B) and flavonol content measurement (C) are performed. Each column represents mean values with SD bar from five biological replicates for each sample. The significant difference is calculated by Duncan statistical analysis at the level of $P \leq 0.05$.

\section{MATERIALS AND METHODS}

\section{Plant Materials}

Plants of E. sagittatum were originally collected from different regions of China and grown in the experimental field of the Epimedium repository at Wuhan Botanical Garden, in the central of China. Nicotiana tabacum cv. NC89 was used for genetic transformation, and transformed tobacco plants were grown in a greenhouse until required.

\section{Isolation of the Full-Length cDNA and Genomic Clones of EsMYBF1}

A survey had been previously investigated that the correlation of gene-to-metabolite during the leaf development stages of E. sagittatum was analyzed (Huang et al., 2015). We found that a candidate gene, encoding a $M Y B$ transcription factor, basically corresponded to the accumulation patterns of the main four BCs (epimedin A, B, C and icariin) and co-expressed with several genes of the flavonol biosynthetic pathway. Therefore, this gene was firstly considered to be a strong candidate gene responsible for the flavonol-derived BC synthesis. In order to obtain the full-length cDNA clone of this gene, both $5^{\prime}$ RACE and $3^{\prime}$-RACE techniques were carried out, following the protocol of SMART RACE cDNA Amplication kit (Clontech, Japan). The expected $5^{\prime}$-RACE and $3^{\prime}$-RACE PCR products were ligated into the pMD19-T vector (Takara, Japan) and sequenced. The assembled sequence was predicted to have an entire ORF, encoding a R2R3-MYB protein. Finally, the full-length cDNA clone of this $M Y B$ gene was isolated from E. sagittatum cDNA template using primers listed in Supplementary Table S1 and PrimeSTAR HS DNA Polymerase (Takara, Japan). This MYB gene of Epimedium revealed a high level of similarity with grape $V v M Y B F 1$ regulator, and thus designated as EsMYBF1. The genomic DNA sequence of $E S M Y B F 1$ gene was also isolated from genomic DNA of E. sagittatum leaves using the same primers for cDNA amplification. The cDNA and genomic sequences of EsMYBF1 have been deposited in GenBank database with the accession number KU365319 and KU365320, respectively.

\section{Sequence Alignment and Phylogenetic Analysis}

The deduced amino acid sequences of EsMYBF1 and other selected $M Y B$ genes were collected from GenBank database, and their accession numbers were indicated in Figure 1. Overall protein sequences of these $M Y B$ genes were used for multiple alignment and phylogenetic analysis. Clustal W program (Thompson et al., 1994) was used to perform the 


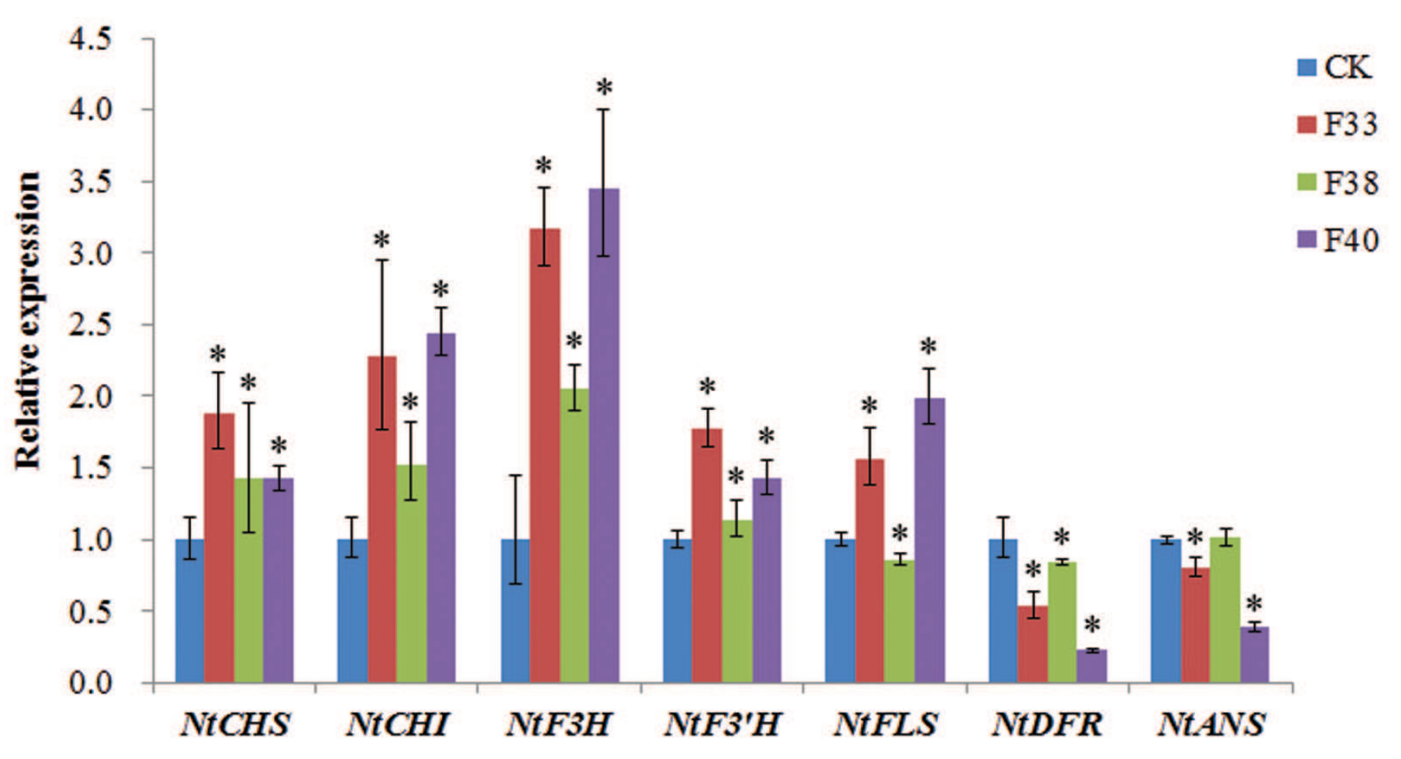

FIGURE 7 | Quantitative PCR assay of the flavonoid pathway genes in transgenic tobacco carrying EsMYBF1 gene. Transcript levels of seven structural genes of flavonoid biosynthetic pathway are determined by quantitative PCR assay, including NtCHS, NtCHI, NtF3H, NtF3' H, NtFLS, NtDFR, and NtANS. Tobacco Tub1 gene is used as an internal control, and comparative Ct method is used to determine the relative expression level. The columns represent average value with SD bar from three technical replicates. Three ESMYBF1 overexpressing tobacco lines (F33, F38, F40) and transgenic tobacco plants carrying the empty vector (CK) are used for qPCR assay. The significant difference of the three overexpression lines against the control line was analyzed using LSD test at the level of $P \leq 0.05$. The * symbol represents the significant difference at the level of $P \leq 0.05$.

multiple alignment analysis, and BioEdit software (version 7.1) was then used for further edition of aligned sequences. Phylogenetic tree was constructed using the neighbor-joining method through MEGA 5 software (Tamura et al., 2011). Default parameters for Clustal W and MEGA 5 softwares were used, unless otherwise mentioned. In addition, the exon/intron genomic structure of EsMYBF1 gene was analyzed using Spidey tool $^{1}$.

\section{Quantitative RT-PCR Assay}

Quantitative RT-PCR (qRT-PCR) assay was carried out to determine the expression levels of EsMYBF1 gene in various tissues of Epimedium plants and the flavonoid biosynthetic pathway genes in transgenic tobacco. Total RNA was extracted using RNAiso Plus (Takara, Japan) from leaves, flower buds and flowers of Epimedium, and from flowers of transgenic tobacco. While RNAiso Plus reagent and Fruit-mate for RNA Purification (Takara, Japan) were used together to extract total RNA from fruits and roots of Epimedium. One microgram of total RNA was firstly digested with gDNA eraser (Takara, Japan) to remove any contaminated genomic DNA, and then was reverse transcribed with PrimeScript RT reagent kit (Takara, Japan) following the supplier's instruction. The reverse transcribed cDNA product was diluted fivefold as the template in qPCR assay. The qPCR assay was set up using SYBR Premix Ex Taq II (Tli RNaseH Plus) kit (Takara, Japan), and run in an ABI7500 Fast Real-Time PCR equipment (Applied Biosystems, USA) following the manufacturer's recommendation. The primers used

${ }^{1}$ http://www.ncbi.nlm.nih.gov/spidey/ for qPCR assay are listed in Supplementary Tables S1 and S2. The melting curve program was included at the end of qPCR program to ensure the specific amplification. The comparative $\mathrm{Ct}$ method $\left(2^{-\Delta \Delta C T}\right)$ was used to determine the relative expression levels of genes (Schmittgen and Livak, 2008). In addition, Epimedium Actin gene and tobacco Tubulin gene were used as internal controls for qPCR assay in Epimedium and tobacco, respectively.

\section{Yeast Two-Hybrid Assay}

In order to confirm that EsMYBF1 is cofactor independent, $\mathrm{Y} 2 \mathrm{H}$ assay of EsMYBF1 with two bHLH regulators of the flavonoid pathway was carried out as described previously (Huang et al., 2016). The coding region of EsMYBF1 was subcloned into the pGADT7 and pGBKT7 vectors (Clontech, Japan), generating the EsMYBF1-AD and EsMYBF1-BD constructs, respectively. The primers used for EsMYBF1 construction were listed in Supplementary Table S1. The pGADT7 construct containing the GAL4 activation domain fused with AtTT8 or EsTT8 protein, and the pGBKT7 construct harboring the GAL4 DNA-binding domain fused with AtTT8 or EsTT8 protein which had been developed previously (Huang et al., 2016), were used directly in this study. The different combined constructs were cotransformed into yeast strain AH109 using the LiAc/SS carrier DNA/PEG method (Gietz and Schiestl, 2007). Transformants were sequentially screened on the double (SD/-Trp/-Leu), quadruple (SD/-Trp/-Leu/-Ade/-His) dropout medium and quadruple dropout medium plus with $\mathrm{X}$-Gal substrate. The growth of yeast cells was observed 3-4 days after incubation. Transformed yeast cells containing pGBKT7-53 + pGADT7-T, 
pGBKT7-Lam + pGADT7-T constructs were used as a positive and a negative control, respectively.

\section{Dual Luciferase Reporter Assay}

Transcription activity of EsMYBF1 TF against promoters of the flavonoid biosynthetic genes was performed using a dual luciferase reporter assay of transient transformed leaves of $N$. benthamiana. The $5^{\prime}$-flanking regions of EsCHS, EsF3H, EsFLS, and EsDFR2 genes were isolated by Tail-PCR (thermal asymmetric interlaced PCR) or inverse PCR and sequenced. Upstream regions from the ATG start site of these four genes, EsCHS (718 bp, Accession number: KC335204), EsF3H (1772 bp, Accession number: KU976281), EsFLS (624 bp, Accession number: KU976282), and EsDFR2 (1138 bp, Accession number: KC335206) were amplified from genomic DNA of E. sagittatum and subcloned into the transient expression reporter vector pGreenII 0800-LUC which contains the CaMV 35S promoterREN cassette and the promoterless-LUC cassette (Hellens et al., 2005). Meantime, the coding regions of EsMYBF1 and AtMYB12 (Accession number: NM_130314.3) were transferred from pMD19-T vector (Takara, Japan) into the transient expression effector vector pGreenII 62-SK which contains the CaMV $35 \mathrm{~S}$ promoter-MCS-CaMV terminator cassette (Hellens et al., 2005). All primers used for reporter and effector constructions were listed in Supplementary Table S1. In addition, the reporter constructs containing EsDFR1 or EsANS promoters and the effector constructs containing EsTT8 or AtTT8 TFs had been developed previously (Huang et al., 2013), and were also directly used for transient expression assay in this study.

All the reporter and effector constructs were transformed into Agrobacterium tumefaciens GV3101 by electroporation method. Agrobacterium was cultured on LB agar supplemented with selection antibiotics and incubated at $28^{\circ} \mathrm{C}$ for 2 days. The confluent bacteria was re-suspended in infiltration buffer (10 $\mathrm{mM} \mathrm{MgCl} 2,0.5 \mu \mathrm{M}$ acetosyringone) to an $\mathrm{OD}_{600}$ of $0.2-0.3$, and incubated at room temperature without shaking for $2-3 \mathrm{~h}$ before infiltration. Transient transformation was conducted by mixing $100 \mu \mathrm{L}$ of Agrobacterium culture transformed with the reporter cassette and $450 \mu \mathrm{L}$ of a second Agrobacterium cultures transformed with the effector cassette containing EsMYBF1, and EsTT8 or AtTT8 TFs. Approximately $300 \mu \mathrm{L}$ of this Agrobacterium mixture was infiltrated into 3-4 young leaves of each plant, with at least two points for each leaf. At least four plants were used for each treatment. The transient expression was assayed 3 days after inoculation.

Dual luciferase assay of transient transformed $N$. benthamiana leaves was carried out according to a previous report (Hellens et al., 2005) using the Dual Luciferase Reporter Assay System (Promega, USA). In brief, $1 \mathrm{~cm}$ leaf disks were punched 3 days after inoculation, and ground in $500 \mu \mathrm{L}$ of Passive Lysis Buffer. $10 \mu \mathrm{L}$ of a $1 / 100$ dilution of this crude extract was measured in $40 \mu \mathrm{L}$ of Luciferase Assay Buffer. Another $40 \mu \mathrm{L}$ of Stop and Glow buffer was then added and a second chemiluminescence measurement was made. Luminescence units were measured using a GloMax 20/20 luminometer (Promega,
USA), with a $5 \mathrm{~s}$ delay and $10 \mathrm{~s}$ integrated measurement. Activity data were expressed as the ratio of LUC to REN activity. Background controls were run with only the promoter-LUC reporter construct (no TF). In some cases, positive controls were run using the AtMYB12 TF with known activity.

\section{Overexpression Vector Construct and Tobacco Transformation}

For ectopic expression of EsMYBF1 in tobacco plants, the full-length cDNA of EsMYBF1 was subcloned from the pMD19-T vector (Takara, Japan) digested with Sal I and Sac I to the modified binary vector pMV (derived from the pBI121 binary vector) digested with Xho I and Sac I, generating the pMV-EsMYBF1 overexpression construct. Thus, EsMYBF1 is expressed under the control of the CaMV $35 \mathrm{~S}$ promoter. This overexpression construct was introduced into Agrobacterium tumefaciens strain EHA105 by electroporation for tobacco transformation. The leaf disk method was used as previously described (Horsch et al., 1985) for genetic transformation of tobacco. Transformed tobacco plants were screened using kanamycin antibiotics as a plant selective marker, and the presence of the introduced EsMYBF1 in transgenic tobacco was confirmed by PCR assay. Finally, three representative independent $\mathrm{T}_{2}$ transgenic lines showing obvious color change of tobacco flowers were used for further analysis. The transgenic tobacco carrying the pMV empty vector was used as the negative control.

\section{Flavonoid Content Measurement in Transgenic Tobacco Flowers}

To evaluate the effects of EsMYBF1 overexpression on flavonoid content in transgenic tobacco, the flavonoid extraction and content measurement are carried out as previously described (Huang et al., 2015). Firstly, flower samples of transgenic tobacco were collected at the blooming stage, frozen immediately in liquid nitrogen and stored at $-70^{\circ} \mathrm{C}$ until use. Total anthocyanin content was measured using a spectrophotometric method, while flavonol content was determined using a HPLC (High Performance Liquid Chromatography) method. In brief, total anthocyanin was extracted using $1 \% \mathrm{HCl} /$ methanol in the dark at $4^{\circ} \mathrm{C}$ overnight with occasional shaking. The extracts were centrifuged at $12,000 \mathrm{~g}$ for $10 \mathrm{~min}$, and the supernatant was filtered with a $0.22 \mu \mathrm{m}$ filter membrane and measured at 530 and $657 \mathrm{~nm}$ for absorbance determination. The equation A530-0.25 $\times$ A657 was used to compensate for the absorption of chlorophyll and its degradation products at $530 \mathrm{~nm}$. Total anthocyanin content was calculated using the subtracted absorbance/fresh weight. Meanwhile, flower powders were immersed into $80 \%$ methanol, and sonicated for $30 \mathrm{~min}$ and then kept at $4^{\circ} \mathrm{C}$ overnight to extract flavonols. Flavonol content in tobacco was calculated as aglycones by preparing acid-hydrolyzed extracts. An aliquot of $400 \mu \mathrm{L}$ of the supernatant was transferred to a fresh tube, acidhydrolyzed by adding $120 \mu \mathrm{L}$ of $3 \mathrm{~N} \mathrm{HCl}$, incubated at $90^{\circ} \mathrm{C}$ for $1 \mathrm{~h}$, and then mixed with $200 \mu \mathrm{L}$ of methanol. This 
hydrolyzation solution was filtered through a $0.22 \mu \mathrm{m}$ filter membrane prior to injection. An Agilent 1100 series HPLC system equipped with an Agilent TC-C18 column (5 $\mu \mathrm{m}$, $4.6 \mathrm{~mm} \times 250 \mathrm{~mm}$ ) was used for chromatographic analysis. The mobile phase of HPLC system consisted of solvent A $(0.1 \%$ formic acid in water), solvent B (acetonitrile) and solvent $\mathrm{C}$ (methanol). The gradient elution program was: $0 \mathrm{~min}, 10 \% \mathrm{~B}+2 \% \mathrm{C} ; 10 \mathrm{~min}, 20 \% \mathrm{~B}+4 \% \mathrm{C} ; 15 \mathrm{~min}$, $50 \% \mathrm{~B}+10 \% \mathrm{C} ; 20 \mathrm{~min}, 20 \% \mathrm{~B}+4 \% \mathrm{C} ; 25 \mathrm{~min}$, $10 \% \mathrm{~B}+2 \% \mathrm{C} ; 28 \mathrm{~min}, 10 \% \mathrm{~B}+2 \% \mathrm{C}$. The column was maintained at $25^{\circ} \mathrm{C}$ and the solvent flow rate was $1.0 \mathrm{~mL} / \mathrm{min}$, and the injection volume was $10 \mu \mathrm{L}$. The detection wavelength was set at $350 \mathrm{~nm}$ for kaempferol and quercetin flavonols. Flavonol compounds were identified with reference to commercial standards of kaempferol and quercetin, and their quantification was measured according to the standard curve of each reference. The flavonoid analysis of each sample was repeated three times using five independent biological replicates.

\section{REFERENCES}

Bogs, J., Jaffé, F. W., Takos, A. M., Walker, A. R., and Robinson, S. P. (2007). The grapevine transcription factor VvMYBPAl regulates proanthocyanidin synthesis during fruit development. Plant Physiol. 143, 1347-1361. doi: 10.1104/pp.106.093203

Borevitz, J. O., Xia, Y., Blount, J., Dixon, R. A., and Lamb, C. (2000). Activation tagging identifies a conserved MYB regulator of phenylpropanoid biosynthesis. Plant Cell 12, 2383-2393. doi: 10.1105/tpc.12.12.2383

Czemmel, S., Stracke, R., Weisshaar, B., Cordon, N., Harris, N. N., Walker, A. R., et al. (2009). The grapevine R2R3-MYB transcription factor VvMYBF1 regulates flavonol synthesis in developing grape berries. Plant Physiol. 151, 1513-1530. doi: 10.1104/pp.109.142059

Gietz, R. D., and Schiestl, R. H. (2007). High-efficiency yeast transformation using the LiAc/SS carrier DNA/PEG method. Nat. Protocols 2, 31-34. doi: 10.1038/nprot.2007.13

Gonzalez, A., Zhao, M., Leavitt, J. M., and Lloyd, A. M. (2008). Regulation of the anthocyanin biosynthetic pathway by the TTG1/bHLH/Myb transcriptional complex in Arabidopsis seedlings. Plant J. 53, 814-827. doi: 10.1111/j.1365313X.2007.03373.x

Havsteen, B. H. (2002). The biochemistry and medical significance of the flavonoids. Pharmacol. Ther. 96, 67-202. doi: 10.1016/S0163-7258(02)00298-X

Hellens, R. P., Allan, A. C., Friel, E. N., Bolitho, K., Grafton, K., Templeton, M. D., et al. (2005). Transient expression vectors for functional genomics, quantification of promoter activity and RNA silencing in plants. Plant Methods 1, 13-26. doi: 10.1186/1746-4811-1-13

Hichri, I., Barrieu, F., Bogs, J., Kappel, C., Delrot, S., and Lauvergeat, V. (2011). Recent advances in the transcriptional regulation of the flavonoid biosynthetic pathway. J. Exp. Bot. 62, 2465-2483. doi: 10.1093/jxb/erq442

Horsch, R., Fry, J., Hoffmann, N., Eichholtz, D., Rogers, S. G., and Fraley, R. (1985). A simple and general method for transferring genes into plants. Science 227, 1229-1231. doi: 10.1126/science.227.4691.1229

Huang, W., Khaldun, A. B. M., Lv, H., Du, L., Zhang, C., and Wang, Y. (2016). Isolation and functional characterization of a R2R3-MYB regulator of the anthocyanin biosynthetic pathway from Epimedium sagittatum. Plant Cell Rep. 35, 883-894. doi: 10.1007/s00299-015-1929-z

Huang, W., Sun, W., Lv, H., Luo, M., Zeng, S., Pattanaik, S., et al. (2013). A R2R3MYB transcription factor from Epimedium sagittatum regulates the flavonoid biosynthetic pathway. PLoS ONE 8:e70778. doi: 10.1371/journal.pone.0070778

Huang, W., Zeng, S., Xiao, G., Wei, G., Liao, S., Chen, J., et al. (2015). Elucidating the biosynthetic and regulatory mechanisms of flavonoid-derived bioactive components in Epimedium sagittatum. Front. Plant Sci. 6:689. doi: 10.3389/fpls.2015.00689

\section{AUTHOR CONTRIBUTIONS}

WH and YW initiated and designed the research. WH, AK, JC performed and analyzed the experiments. CZ and HL contributed to reagents, materials and analysis tools. WH wrote the paper, LY and YW revised the paper.

\section{ACKNOWLEDGMENTS}

This study was supported by funds of National Natural Science Foundation of China (31270340, 31200225). We thank Dr. Wei Sun at Institute of Chinese Materia Medica for critical editing.

\section{SUPPLEMENTARY MATERIAL}

The Supplementary Material for this article can be found online at: http://journal.frontiersin.org/article/10.3389/fpls.2016.01089

Jiang, J., Song, J., and Jia, X.-B. (2015). Phytochemistry and ethnopharmacology of Epimedium L. Species. Chin. Herb. Med. 7, 204-222. doi: 10.1016/S16746384(15)60043-0

Jiang, J., Zhao, B.-J., Song, J., and Jia, X.-B. (2016). Pharmacology and clinical application of plants in Epimedium L. Chin. Herb. Med. 8, 12-23. doi: 10.1016/S1674-6384(16)60003-5

Kobayashi, S., Goto-Yamamoto, N., and Hirochika, H. (2004). Retrotransposoninduced mutations in grape skin color. Science 304, 982. doi: $10.1126 /$ science. 1095011

Koes, R., Verweij, W., and Quattrocchio, F. (2005). Flavonoids: a colorful model for the regulation and evolution of biochemical pathways. Trends Plant Sci. 10, 236-242. doi: 10.1016/j.tplants.2005.03.002

Li, C., Li, Q., Mei, Q., and Lu, T. (2015a). Pharmacological effects and pharmacokinetic properties of icariin, the major bioactive component in Herba Epimedii. Life Sci. 126, 57-68. doi: 10.1016/j.lfs.2015.01.006

Li, Y., Chen, M., Wang, S., Ning, J., Ding, X., and Chu, Z. (2015b). AtMYB11 regulates caffeoylquinic acid and flavonol synthesis in tomato and tobacco. Plant Cell Tiss. Organ Cult. 122, 309-319. doi: 10.1007/s11240-015-0767-6

Li, Z., Xu, Y., Wang, Y., and Huang, H. (2005). Status and prospect of research on medicinal plants of Epimedium L. Zhong Cao Yao 36, 289-295.

Liu, J., Osbourn, A., and Ma, P. (2015). MYB transcription factors as regulators of phenylpropanoid metabolism in plants. Mol. Plant 8, 689-708. doi: 10.1016/j.molp.2015.03.012

Luo, J., Butelli, E., Hill, L., Parr, A., Niggeweg, R., Bailey, P., et al. (2008). AtMYB12 regulates caffeoyl quinic acid and flavonol synthesis in tomato: expression in fruit results in very high levels of both types of polyphenol. Plant J. 56, 316-326. doi: 10.1111/j.1365-313X.2008.03597.x

Ma, H., He, X., Yang, Y., Li, M., Hao, D., and Jia, Z. (2011). The genus Epimedium: an ethnopharmacological and phytochemical review. J. Ethnopharmacol. 134, 519-541. doi: 10.1016/j.jep.2011.01.001

Mehrtens, F., Kranz, H., Bednarek, P., and Weisshaar, B. (2005). The Arabidopsis transcription factor MYB12 is a flavonol-specific regulator of phenylpropanoid biosynthesis. Plant Physiol. 138, 1083-1096. doi: 10.1104/pp.104.058032

Misra, P., Pandey, A., Tiwari, M., Chandrashekar, K., Sidhu, O. P., Asif, M. H., et al. (2010). Modulation of transcriptome and metabolome of tobacco by Arabidopsis transcription factor, AtMYB12, leads to insect resistance. Plant Physiol. 152, 2258-2268. doi: 10.1104/pp.109.150979

Nesi, N., Jond, C., Debeaujon, I., Caboche, M., and Lepiniec, L. (2001). The Arabidopsis TT2 gene encodes an R2R3 MYB domain protein that acts as a key determinant for proanthocyanidin accumulation in developing seed. Plant Cell 13, 2099-2114. doi: 10.1105/tpc.13.9.2099

Pandey, A., Misra, P., Choudhary, D., Yadav, R., Goel, R., Bhambhani, S., et al. (2015a). AtMYB12 expression in tomato leads to large scale differential 
modulation in transcriptome and flavonoid content in leaf and fruit tissues. Sci. Rep. 5, 12412. doi: 10.1038/srep 12412

Pandey, A., Misra, P., and Trivedi, P. K. (2015b). Constitutive expression of Arabidopsis MYB transcription factor, AtMYB11, in tobacco modulates flavonoid biosynthesis in favor of flavonol accumulation. Plant Cell Rep. 34, 1515-1528. doi: 10.1007/s00299-015-1803-z

Ramsay, N. A., and Glover, B. J. (2005). MYB-bHLH-WD40 protein complex and the evolution of cellular diversity. Trends Plant Sci. 10, 63-70. doi: 10.1016/j.tplants.2004.12.011

Schmittgen, T. D., and Livak, K. J. (2008). Analyzing real-time PCR data by the comparative CT method. Nat. Protoc. 3, 1101-1108. doi: 10.1038/nprot.2008.73

Stracke, R., Ishihara, H., Huep, G., Barsch, A., Mehrtens, F., Niehaus, K., et al. (2007). Differential regulation of closely related R2R3-MYB transcription factors controls flavonol accumulation in different parts of the Arabidopsis thaliana seedling. Plant J. 50, 660-677. doi: 10.1111/j.1365-313X.2007.03078.x

Tamura, K., Peterson, D., Peterson, N., Stecher, G., Nei, M., and Kumar, S. (2011). MEGA5: molecular evolutionary genetics analysis using maximum likelihood, evolutionary distance, and maximum parsimony methods. Mol. Biol. Evol. 28, 2731-2739. doi: 10.1093/molbev/msr121

Terrier, N., Torregrosa, L., Ageorges, A., Vialet, S., Verries, C., Cheynier, V., et al. (2009). Ectopic expression of VvMybPA2 promotes proanthocyanidin biosynthesis in grapevine and suggests additional targets in the pathway. Plant Physiol. 149, 1028-1041. doi: 10.1104/pp.108.131862

Thompson, J. D., Higgins, D. G., and Gibson, T. J. (1994). CLUSTAL W: improving the sensitivity of progressive multiple sequence alignment through sequence weighting, position-specific gap penalties and weight matrix choice. Nucleic Acids Res. 22, 4673-4680. doi: 10.1093/nar/22.22.4673

Walker, A. R., Lee, E., Bogs, J., Mcdavid, D. A. J., Thomas, M. R., and Robinson, S. P. (2007). White grapes arose through the mutation of two similar and adjacent regulatory genes. Plant J. 49, 772-785. doi: 10.1111/j.1365-313X.2006.02997.x
Winkel-Shirley, B. (2001). Flavonoid biosynthesis. A colorful model for genetics, biochemistry, cell biology, and biotechnology. Plant Physiol. 126, 485-493. doi: 10.1104/pp.126.2.485

Xu, W., Dubos, C., and Lepiniec, L. (2015). Transcriptional control of flavonoid biosynthesis by MYB-bHLH-WDR complexes. Trends Plant Sci. 20, 176-185. doi: 10.1016/j.tplants.2014.12.001

Yao, L. H., Jiang, Y. M., Shi, J., Tomás-Barberán, F. A., Datta, N., Singanusong, R., et al. (2004). Flavonoids in food and their health benefits. Plant Food Hum. Nutr. 59, 113-122. doi: 10.1007/s11130-0040049-7

Zeng, S., Liu, Y., Hu, W., Liu, Y., Shen, X., and Wang, Y. (2013). Integrated transcriptional and phytochemical analyses of the flavonoid biosynthesis pathway in Epimedium. Plant Cell Tiss. Org. Cult. 115, 355-365. doi: 10.1007/s11240-013-0367-2

Zimmermann, I. M., Heim, M. A., Weisshaar, B., and Uhrig, J. F. (2004). Comprehensive identification of Arabidopsis thaliana MYB transcription factors interacting with R/B like BHLH proteins. Plant J. 40, 22-34. doi: 10.1111/j.1365-313X.2004.02183.x

Conflict of Interest Statement: The authors declare that the research was conducted in the absence of any commercial or financial relationships that could be construed as a potential conflict of interest.

Copyright (c) 2016 Huang, Khaldun, Chen, Zhang, Lv, Yuan and Wang. This is an open-access article distributed under the terms of the Creative Commons Attribution License (CC BY). The use, distribution or reproduction in other forums is permitted, provided the original author(s) or licensor are credited and that the original publication in this journal is cited, in accordance with accepted academic practice. No use, distribution or reproduction is permitted which does not comply with these terms. 